\title{
To Study the Correlation of Serum Cholinesterase Level With Different Scoring Systems in Cirrhosis of the Liver
}

\author{
Jagadeesh B S ${ }^{1}$, Ravi K ${ }^{2}$, Avinash H R ${ }^{* 1}$, Nitish Ashok Gurav ${ }^{1}$ \\ ${ }^{1}$ Senior resident, Department of Medicine, Bangalore Medical College and Research Institute, KR Road, \\ Bangalore, Karnataka, India \\ ${ }^{2}$ Professor and Head, Department of Medicine, Bangalore Medical College and Research Institute, KR Road, \\ Bangalore, Karnataka, India.
}

*Corresponding author: Avinash H R; avinashhr19@gmail.com

Received 03 February 2021;

Accepted 16 February 2021;

Published 25 February 2021

\begin{abstract}
Background: Chronic liver disease in the clinical context is a disease process of the liver that involves a process of progressive destruction and regeneration of the liver parenchyma leading to fibrosis and cirrhosis1. The serum cholinesterase is mainly synthesized in the liver and it is released into the blood, which is reduced in liver dysfunction due to reduced synthesis, marked reduction of cholinesterase in liver dysfunction and restoration of synthesis with hepatocyte recovery suggest serum cholinesterase activity might be a more specific marker of liver dysfunction than traditional liver function tests. Objectives: To estimate the level of serum cholinesterase in patients with cirrhosis of the liver.

To correlate the level of serum cholinesterase with different scoring systems of cirrhosis of the liver and assess the utility of serum cholinesterase levels in prognostification. Materials and Methods: A cross-sectional, hospital-based, time-bound study conducted on 200 patients with cirrhosis of liver attending medicine OPD and getting admitted in hospitals attached to BMCRI from November 1 st 2016 to August 30th2018. All cirrhosis of liver patients were included and patients with Pregnancy, Acute infection, Chronic infection like tuberculosis and Oral contraceptive use were excluded. Serum cholinesterase, ultrasound abdomen, prothrombin Time, International Normalized Ratio, liver function test, Child-Pugh score, MELD score were measured. Results: In the study, the majority of the study subjects belonged to the age group $41-50$ years (38.5\%), followed by 31-40 years (21.5\%), $51-60$ years (18.5\%). Sex distribution male $70 \%$ and female $30 \%$. Serum Cholinesterase was positively correlated with Albumin and Prothrombin time and negatively correlated with MELD, Creatinine and Child-Pugh Score. The mean S. cholinesterase values found in study subjects belonged to Child-Pugh Score A, B and C were $4235.17+341.260,3226.26+707.206$ and $1764.09+808.797$. The ANOVA results showed that there was a significant association found between child-pugh scores and S. cholinesterase ( $p-0.001)$. Conclusion: The Study has demonstrated that the level of cholinesterase is correlated with the severity of the liver disease. Serum cholinesterase shows a good correlation with serum albumin, PT INR, Child-pugh score, MELD score. Compared to the above parameters serum cholinesterase is less complex and not easily affected by treatments for decompensated cirrhosis.
\end{abstract}

Keywords: Cirrhosis of liver; Serum cholinesterase; Child-pugh score; MELD

\section{Introduction}

Cirrhosis is defined as a diffuse process with fibrosis and nodule formation. It is the result of the fibrogenesis that occurs with chronic liver injury ${ }^{[1]}$. The causes of cirrhosis are multiple and include metabolic, inflammatory, congenital, and toxic liver diseases ${ }^{[2]}$. The most common causes of cirrhosis are chronic alcoholism and chronic hepatitis C and B, followed by biliary diseases and hemochromatosis ${ }^{[3]}$. Regardless of the cause of cirrhosis, the pathologic features consist of the development of fibrosis to the point that there is architectural distortion with the formation of regenerative nodules ${ }^{[4]}$.
Liver function tests (LFTs) are useful in the evaluation and treatment of patients with hepatic dysfunction, comprising ALT, AST, alkaline phosphatase, bilirubin, albumin and prothrombin time. These tests reveal abnormal results in patients with clinical problems other than liver dysfunction ${ }^{[5]}$.

Serum Cholinesterase is synthesized in the liver and its levels are reduced in cirrhosis of the liver and its levels are not affected by treatment compared to other markers ${ }^{[6]}$.

Cholinesterase is the enzymes classified by Mendel and Rudney into true cholinesterase (acetylcholinesterase) present in erythrocytes and glial tissue and pseudocholinesterase (serum cholinesterase) present in the serum and synthesized in the liver ${ }^{[7]}$. Marked decrease in serum cholinesterase synthesis in liver disease 
suggest that its activity might be a specific indicator of liver dysfunction than other liver function tests ${ }^{[8]}$.

Serum cholinesterase shows a good correlation with serum albumin, PT INR, Child-pugh score and the MELD score. Since it discriminates well between De-compensated Cirrhosis and Compensated Cirrhosis, low levels in cirrhosis may be considered as a prognostic marker of advanced liver disease ${ }^{[9]}$.

To diagnose liver disease a large number of conventional liver function tests are being performed for many years but they don't have $100 \%$ sensitivity, specificity. Many times conventional parameters of liver function tests are raised in nonliver diseases ${ }^{[10]}$.

\section{Aims and objectives}

1. To estimate the level of serum cholinesterase in patients with cirrhosis of the liver.

2. To correlate the level of serum cholinesterase with different scoring systems of cirrhosis of the liver and assess the utility of serum cholinesterase levels in prognostification.

\section{Materials and Methods}

The study was conducted on 200 patients with cirrhosis of liver attending medicine OPD and getting admitted in hospitals attached to BMCRI from November 1st2016 to August 30th, 2018.

\section{Inclusion criteria:}

1. Patients willing to give written informed consent

2. Patients of either sex aged 18years and above.

3. All patients diagnosed to have cirrhosis of the liver clinically and radiologically in hospitals attached to BMCRI.

\section{Exclusion criteria:}

1. Age less than 18 years

2. Pregnancy

3. Acute infection

4. A Chronic infection like tuberculosis

5. Oral contraceptive use

6. Drugs-anticholinesterase-inhibitor, glucocorticoids, esmolol, metoclopramide, cyclophosphamide.

7. Extensive burn injuries.

8. Organophosphate poisoning.
After obtaining clearance and approval from the institutional ethics committee and written informed consent, the patients with cirrhosis of liver fulfilling inclusion and exclusion criteria will be enrolled in the study.

For each patient the following data will be collected: History, clinical examination, Serum cholinesterase, ultrasound abdomen, prothrombin Time, International Normalized Ratio, liver function test, Child-Pugh score, MELD score.

Sample size: 200 cases. Calculated using the following

Formula $\mathrm{N}=\mathrm{Z} 2$ pqd Where, $\mathrm{N}=$ Sample size

$\mathrm{Z} 2$ at $95 \%$ confidence interval $=1.96$

$\mathrm{p}=0.6151-\mathrm{p}=\mathrm{q}=0.38 \mathrm{~d}^{2}=0.10$

With $10 \%$ absolute precision $(\alpha)$ at $95 \%$ confidence interval, sample size $(\mathrm{N})$ is 180 .

Expecting the $10 \%$ non-response, the final sample size of 200 will be taken for the study.

\section{Statistical Analysis}

Categorical variables will be analyzed using descriptive statistics. ANOVA test was used to compare 3 or more groups. Pearson correlation test used to correlate quantitative variable. The $\mathrm{P}$ values $<0.05$ were considered statistically significant.

\section{Results and Analysis}

In the study, the majority of the study subjects belonged to the age group $41-50$ years $(38.5 \%)$, followed by $31-40$ years $(21.5 \%), 51$ -60 years $(18.5 \%)$. Sex distribution male $70 \%$ and female $30 \%$

Table1: Etiology of cirrhosis of the liver

\begin{tabular}{|l|l|l|}
\hline Etiology & Frequency & Percent \\
\hline Alcohol & 176 & 88 \\
\hline HBsAg & 14 & 7 \\
\hline HCV & 3 & 1.5 \\
\hline NASH & 7 & 3.5 \\
\hline
\end{tabular}

Alcohol is the most common etiology present in $88 \%$ of cases followed by HBsAg in 7\%, NASH in $3.5 \%$ and $\mathrm{HCV}$ in $1.5 \%$ of cases.

Alcohol consumption was given to $88 \%$ of the study subjects. Nonalcoholics were $12 \%$.

Child-Pugh Class(CPS)

The Majority of the study subjects belonged to Child-Pugh class 'C' (76\%), followed by 'B' (21\%) and 'A' (3\%).

\section{Methodology}

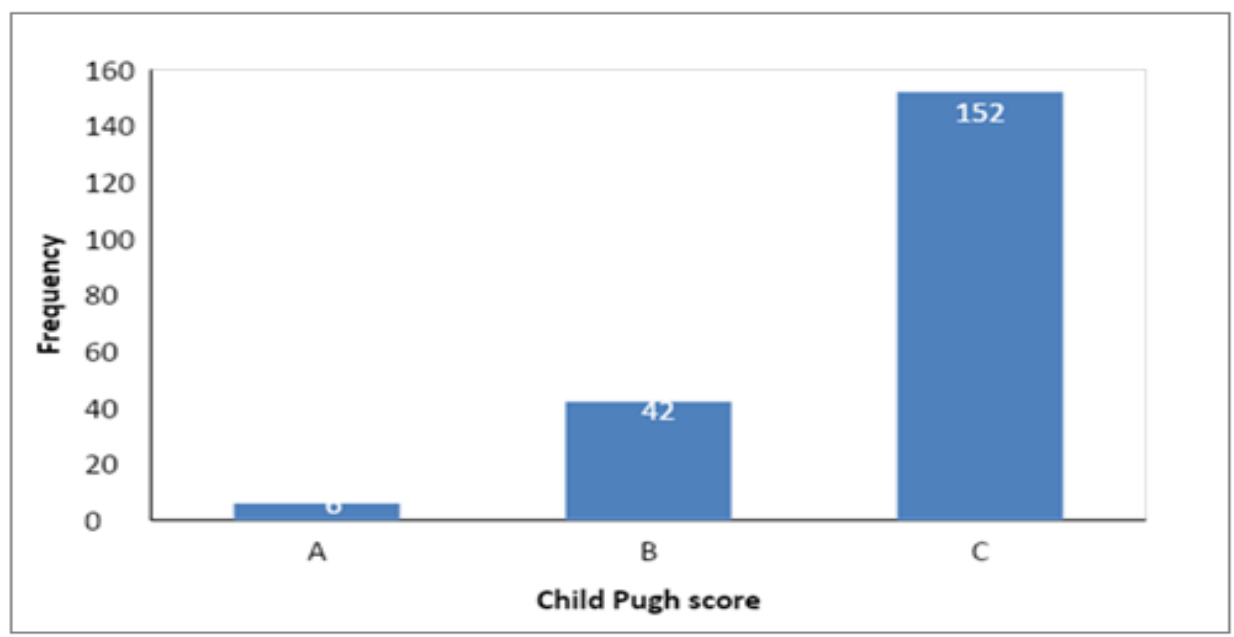

Figure 1: CPS 
Table 2: Distribution of Parameters in the study

\begin{tabular}{|l|l|l|l|l|}
\hline & Mean & SD & Minimum & Maximum \\
\hline $\mathrm{Hb}(\mathrm{gm} / \mathrm{dl})$ & 9.70 & 2.30 & 2.40 & 16.40 \\
\hline TLC(/cumm) & 10834.14 & 6613.41 & 21.0 & 39834.14 \\
\hline PLT(lakh/cumm) & 1.34 & 0.82 & 0.17 & 5.72 \\
\hline Creatinine & 1.46 & 1.41 & 0.20 & 8.50 \\
\hline T.Bilirubin & 7.64 & 8.32 & 0,20 & 36.90 \\
\hline S.Albumin & 2.42 & 0.18 & 1.30 & 4.90 \\
\hline PT & 20.91 & 9.64 & 9.20 & 90.84 \\
\hline INR & 1.93 & 1.29 & 0.35 & 13 \\
\hline
\end{tabular}

Table 3.correlation of different variables with serum cholinesterase

\begin{tabular}{|l|l|l|l|l|l|}
\hline S. Cholinesterase & MELD & ALBUMIN & CREATININE & CP Score & Prothrombin time \\
\hline Pearson Correlation & $-.517^{* * *}$ & $.596^{* * *}$ & $-.203^{* *}$ & -0.740 & 0.424 \\
\hline Sig. (2-tailed) & .000 & .000 & .004 & 0.000 & 0.000 \\
\hline
\end{tabular}

S. Cholinesterase was positively correlated with Albumin $(r-0.596)$ and Prothrombin time $(r-0.424)$ and negatively correlated with MELD ( $r$ -0.517), Creatinine ( $r-0.203)$ and CP Score $(r--0.740)$. All the variables were significantly correlated with $S$. cholinesterase ( $p<0.05)$

Table no 4: Serum cholinesterase in alcoholic and non alcoholic liver cirrhosis

\begin{tabular}{|l|l|l|l|l|l|}
\hline \multirow{2}{*}{ Alcoholic history } & \multirow{2}{*}{$\mathbf{N}$} & S. Cholinesterase & \multirow{2}{*}{ t-test } & \multirow{2}{*}{ p-value } \\
\cline { 3 - 5 } & & Mean & Std. Deviation & 0.435 & 0.664 \\
\hline Alcoholic & 176 & 2157.18 & 1071.549 & 836.398 & \\
\hline Nonalcoholic & 24 & 2058.00 & & \\
\hline
\end{tabular}

The mean S. Cholinesterase in alcoholic and nonalcoholic was $2157.18+1071.549$ and $2058+836.398$. The t-test value shows that there was no significant difference between alcoholic history and $S$. Cholinesterase $(\mathrm{p}-0.664)$.

Table 5: Correlation of serum cholinesterase and child-pugh score

\begin{tabular}{|l|l|l|l|l|}
\hline & Mean & Std. Deviation & t-test & p-value \\
\cline { 1 - 3 } CP SCORE & 11.52 & 2.429 & 28.885 & 0.001 \\
\end{tabular}

The mean CP score was $11.52+2.429$ and the mean $\mathrm{S}$. Cholinesterase was $2145.28+1044.814$.The t-test value shows that there was a significant difference between CP Score and S. Cholinesterase $(p-0.001)$.

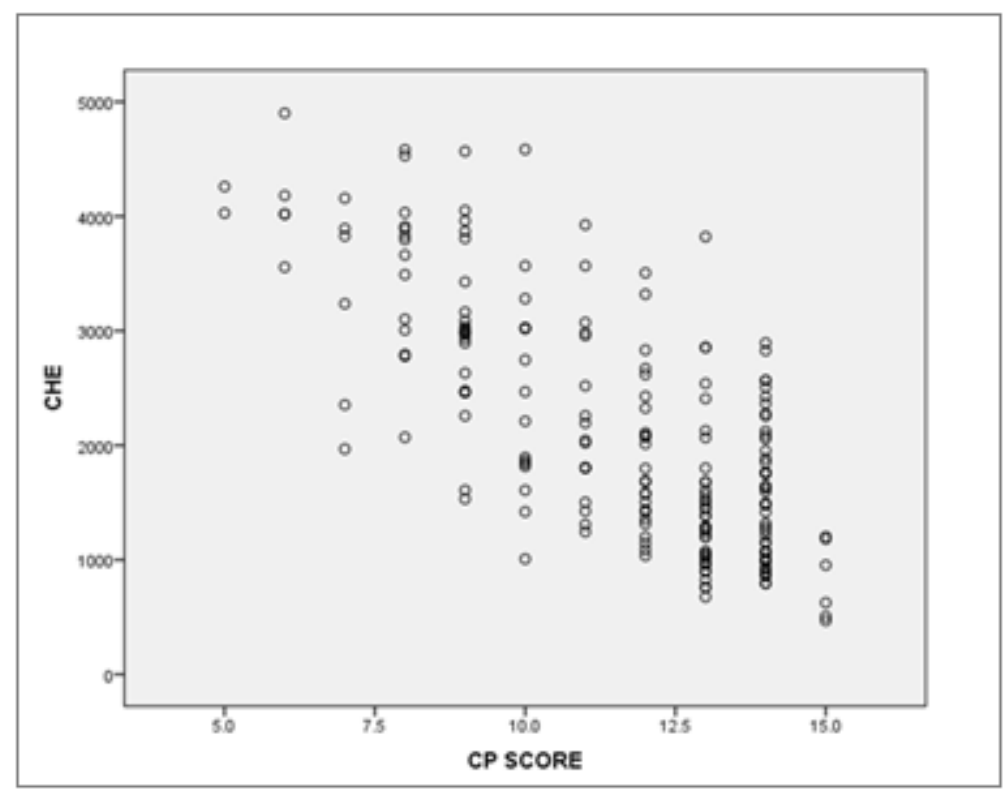

Figure 2: correlation of serum cholinesterase and child-pugh score

Table 6: Correlation of serum cholinesterase and child-pugh class

\begin{tabular}{|l|l|l|l|l|l|l|l|}
\hline \multirow{2}{*}{ Child-Pugh score } & \multirow{2}{*}{$\mathbf{N}$} & \multicolumn{2}{|l|}{ S. Cholinesterase } & \multirow{2}{*}{ Minimum } & \multirow{2}{*}{ Maximum } & \multirow{2}{*}{ F-value } & \multirow{2}{*}{ p-value } \\
\cline { 3 - 6 } & & Mean & Std. Deviation & & 40.015 & 0.001 \\
A & 6 & 4235.17 & 341.260 & 4019 & 4901 & \\
\hline B & 42 & 3226.26 & 707.206 & 1532 & 4582 & \\
\hline C & 152 & 1764.09 & 808.797 & 468 & 4584 & \\
\hline Total & 200 & 2145.28 & 1044.814 & 468 & 4901 & \\
\hline
\end{tabular}


The mean S. cholinesterase values found in study subjects belonged to Child-Pugh Score A, B and C were $4235.17+341.260,3226.26+$ 707.206 and 1764.09+808.797. The ANOVA results showed that there was significant association found between child-pugh scores and S. cholinesterase $(\mathrm{p}-0.001)$

Table 7: Correlation of serum cholinesterase and meld score

\begin{tabular}{|l|l|l|l|l|}
\hline & Mean & Std. Deviation & t-test & p-value \\
\cline { 1 - 2 } & 21.83 & 9.62 & 28.7409 & 0.001 \\
\cline { 1 - 2 } & 2145.28 & 1044.814 & & \\
\end{tabular}

Table 8: Correlation of serum cholinesterase and meld score

\begin{tabular}{|c|c|c|c|c|c|c|c|}
\hline \multirow{2}{*}{ MELD } & \multirow[b]{2}{*}{$\mathbf{N}$} & \multicolumn{2}{|c|}{ S. Cholinesterase } & \multirow[b]{2}{*}{ Minimum } & \multirow{2}{*}{ Maximum } & \multirow[b]{2}{*}{ F-value } & \multirow[b]{2}{*}{ P-value } \\
\hline & & Mean & Std. Deviation & & & & \\
\hline$<10$ & 12 & 3610.67 & 809.470 & 1968 & 4901 & \multirow[t]{4}{*}{36.516} & \multirow[t]{4}{*}{0.000} \\
\hline $10-20$ & 90 & 2477.79 & 954.047 & 871 & 4584 & & \\
\hline$>20$ & 98 & 1660.47 & 851.117 & 468 & 4568 & & \\
\hline Total & 200 & 2145.28 & 1044.814 & 468 & 4901 & & \\
\hline
\end{tabular}

The mean MELD was $21.83+9.62$ and the mean S. Cholinesterase was $2145.28+1044.814$. The $t$ test value shows that there was a significant difference between MELD and S. Cholinesterase ( $\mathrm{p}-0.001)$.

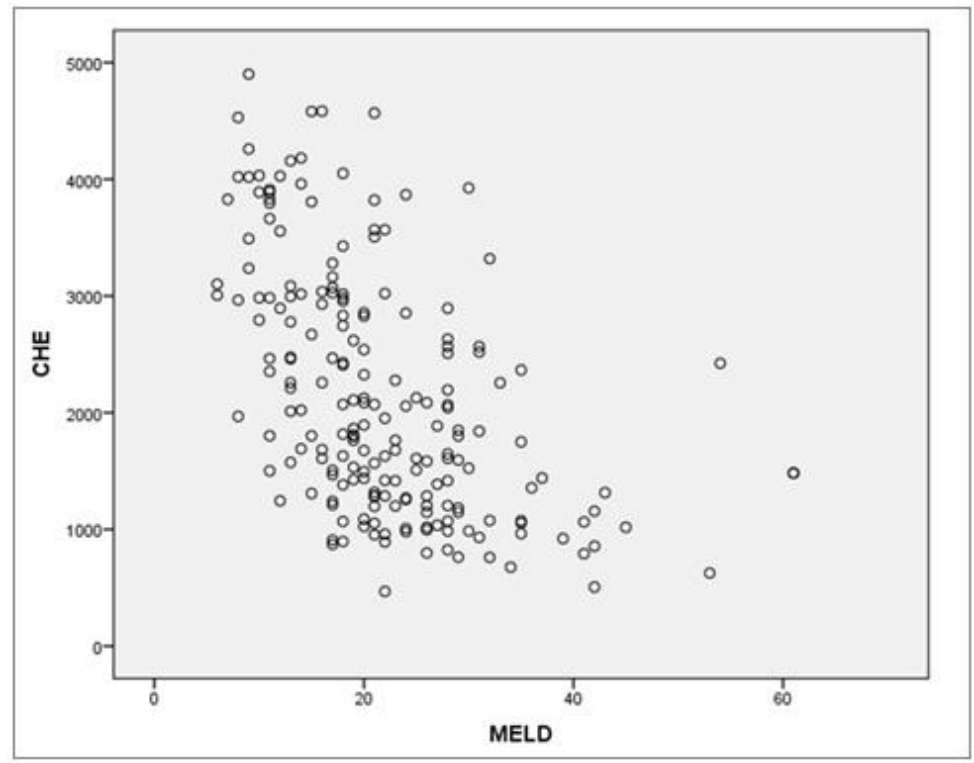

Figure 3: Correlation of serum cholinesterase and meld score

The mean S. cholinesterase value was found in study subjects with a MELD score of less than 10 was $3610.67+809.47$, followed by a MELD score of $10-20(2477.79+954.047)$ and $>20(1660.47+851.117)$. The ANOVA results showed that there was a significant association found between MELD scores and S. cholinesterase $(\mathrm{p}<0.001)$

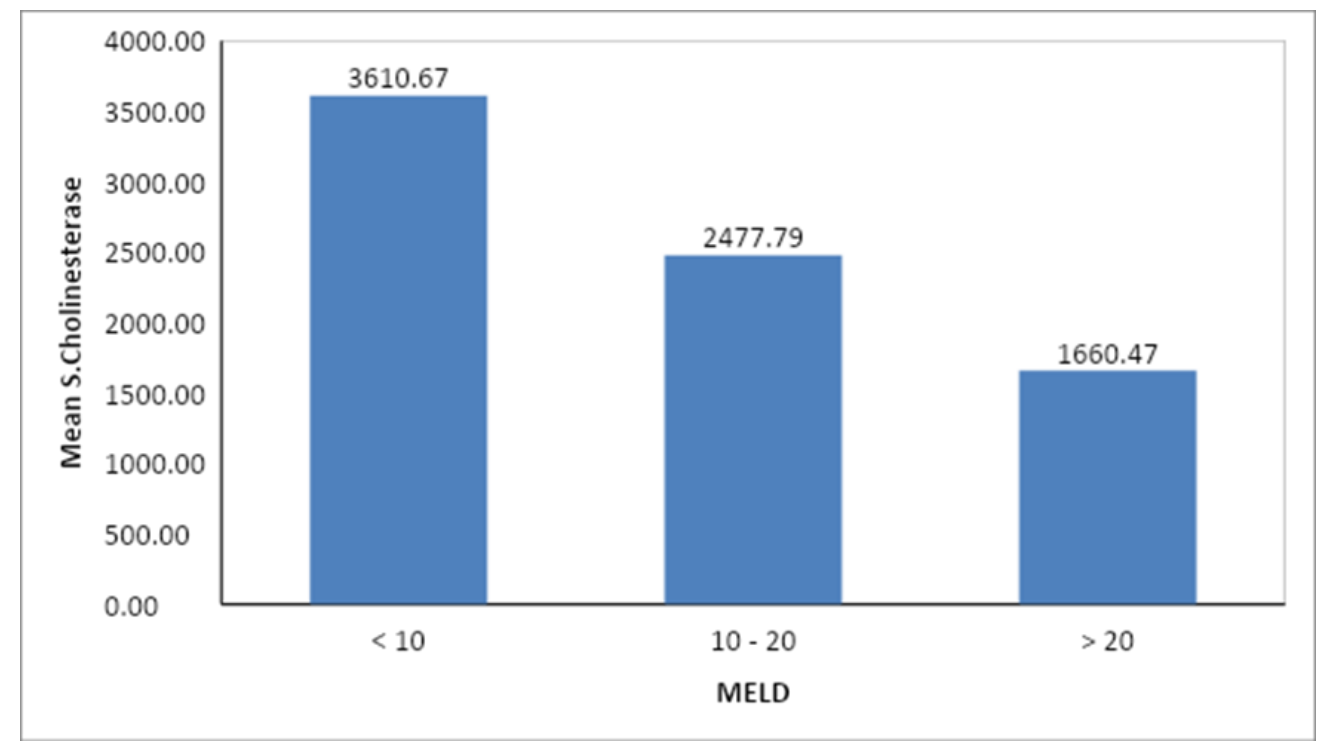

Figure 4: Correlation of serum cholinesterase and meld score 


\section{Discussion}

Chronic liver disease in the clinical context is a disease process of the liver that involves a process of progressive destruction and regeneration of the liver parenchyma leading to fibrosis and cirrhosis ${ }^{[1]}$. The serum cholinesterase is mainly synthesized in the liver and is released into the blood, which is reduced in liver dysfunction due to reduced synthesis, marked reduction of cholinesterase in liver dysfunction and restoration of synthesis with hepatocyte recovery suggest serum cholinesterase activity might be a more specific marker of liver dysfunction than traditional liver function tests.

In our study 200 patients of clinically or radiologically diagnosed cases of cirrhosis were taken and serum cholinesterase was estimated for all patients, which is correlated with child -pugh score and meld score. Among 200 cases 181 patients were male accounting for $91 \%$ of cases and the remaining 19 patients were females accounting for $9.5 \%$ of cases. The Majority of patients belonged to the age group between $41-50$ years.

Out of 200 patients 176 were alcoholics with significant alcohol consumption, which is being the most common cause of cirrhosis accounting for $88 \%$, followed by hepatitis $\mathrm{B}(7 \%)$ and hepatitis $\mathrm{c}(1.5 \%)$. The Majority of patients belonged to child-pugh class $\mathrm{C}$, accounting for $76 \%$ (152) followed by class B accounting for $21 \%$ of cases and $3 \%$ (6) of cases belonged to class A. The mean MELD score in our study was 21.

In this study, the serum cholinesterase levels were decreased in all patients compared to standard value, with a significant $p$-value of $<0.001$. The mean \pm SD of serum cholinesterase in cases was $2145.28 \pm 1044.814$, this is by the study by Jeyamani Ramachandran et al., where serum cholinesterase levels in cirrhotic ranged between 110 to $8143 \mathrm{IU} / \mathrm{l}$ with a median of 1590 IU/L.

In our study there was no statistically significant difference in serum cholinesterase between alcoholic and non-alcoholic patients ( $\mathrm{p}$ - 0.664). Serum cholinesterase was positively correlated with prothrombin time, Total bilirubin and negatively correlated with serum albumin. These results were matched with Jeyamani Ramachandran et al., where $\mathrm{ChE}$ levels reflect the functional integrity of liver.

The mean S. cholinesterase values found in study subjects belonged to Child-Pugh Score A, B and C were $4235.17+$ $341.260,3226.26+707.206$ and $1764.09+808.797$. The ANOVA results showed that there was a significant association found between child-pugh scores and $\mathrm{S}$. cholinesterase $(\mathrm{p}-0.001)$. This by a Fanping Ming et al,. where the patients with child- pugh class a had a cholinesterase value $5368+1657$, class B had $2943+1212$ and class $\mathrm{C}$ had $1832+710$, which showed serum cholinesterase tend to decrease significantly in three grades of cirrhosis.

The mean S. cholinesterase value was found in study subjects with aa MELD score of less than 10 was $3610.67+$ 809.47 , followed by a MELD score of $10-20(2477.79+954.047)$ and $>20(1660.47+851.117)$. The ANOVA results showed that there was a significant association found between MELD scores and $\mathrm{S}$. cholinesterase $(\mathrm{p}<0.001)$. In our study we found a significant correlation between serum cholinesterase and MELD score in cirrhotic patients, where patients with higher MELD scores have a lower level of cholinesterase $(\mathrm{p}-<0.001)$. This by study done by Jayamani Ramachandran et al,,where the patients with MELD score $<15$ had a mean serum cholinesterase of 1940IU/L and patients with MELD score $>15$ had a mean serum cholinesterase of $1084 \mathrm{IU} / \mathrm{L}$ with a significant correlation coefficient $(\mathrm{p}<0.001)$

\section{Conclusion}

In conclusion, the findings of this study have demonstrated that the level of cholinesterase is correlated with the severity of liver disease. It shows a good correlation with serum albumin, PT INR, Child-pugh score, MELD score. Compared to the above parameters serum cholinesterase is less complex and not easily affected by treatments for decompensated cirrhosis. The combination of cholinesterase with above parameters may be more subjective and accurate in evaluating the liver reserve function of cirrhotic patients.

\section{Limitation}

The Sample size was a small and single-center study

\section{References}

[1] Sherlock's disease of liver and biliary system, 12th edition, chapter 7-Hepatic cirrhosis, page no-103.

[2] Schiff's disease of liver, 11th edition, Hepatic fibrosis, 297-311.

[3] Guadalupe GT, Joseph L, Management and Treatment of Patients With Cirrhosis and Portal Hypertension 2009; 104:1802-29

[4] Robbins textbook of basic pathology, 8th edition, 63339.

[5] Meng F, Yin X, Ma X, Guo XD, Jin B, Li H. Assessment of the value of serum cholinesterase as a liver function test for cirrhotic patients. Biomedical reports. 2013 Mar 1;1(2):265

[6] Ramachandran J, Sajith KG, Priya S, Dutta AK, Balasubramanian KA. Serum cholinesterase is an excellent biomarker of liver cirrhosis. Tropical Gastroenterology. 2015 May 16;35(1):15-20.

[7] Mendel B, Rudney H.Studies on cholinesterase: 1.Cholinesterase and psedocholinesterase.Biochem J.1943;37;p59-63.

[8] Miller RD; Miller's Anesthesia, 6th Edition: Philadelphia, Elsevier, Churchill, Livingstone, 2005. pp 487-488.

[9] Ramachandran J, Sajith KG, Priya S, Dutta AK, Balasubramanian KA. Serum cholinesterase is an excellent biomarker of liver cirrhosis. Tropical Gastroenterology. 2015 May 16;35(1):15-20.

[10] Weisinger RA; „Cecil Textbook of Medicineee, 21st edn: Laboratory tests in liver disease and approach to the patient with abnormal test,p.775-777. 